$\mathrm{UCB} / \mathrm{PTH}-02 / 47$

LBNL-51652

\title{
Extra Dimensions at the Weak Scale and Deviations from Newtonian Gravity
}

\author{
Z. Chacko ${ }^{\mathrm{a}, \mathrm{b}, \mathrm{d}}$, and E. Perazzi ${ }^{\mathrm{c}, \mathrm{e}}$ \\ a Department of Physics, University of California, Berkeley, CA 94720, USA \\ b Theoretical Physics Group, Lawrence Berkeley National Laboratory, \\ Berkeley, CA 94720, USA \\ c Physics Department, Sloane Laboratory, \\ Yale University, New Haven, CT 06520, USA \\ d email: zchacko@thsrv.lbl.gov \\ email: elena.perazzi@yale.edu
}

\begin{abstract}
We consider theories in which the Standard Model gauge fields propagate in extra dimensions whose size is around the electroweak scale. The Standard Model quarks and leptons may either be localized to a brane or propagate in the bulk. This class of theories includes models of Scherk-Schwarz supersymmetry breaking and universal extra dimensions. We consider the problem of stabilizing the volume of the extra dimensions. We find that for a large class of stabilization mechanisms the field which corresponds to fluctuations of the volume remains light even after stabilization, and has a mass in the $10^{-3} \mathrm{eV}$ range. In particular this is the case if stabilization does not involve dynamics at scales larger than the cutoff of the higher dimensional Standard Model, and if the effective theory below the compactification scale is four dimensional. The mass of this field is protected against large radiative corrections by the general covariance of the higher dimensional theory and by the weakness of its couplings, which are Planck suppressed. Its couplings to matter mediate forces whose strength is comparable to that of gravity and which can give rise to potentially observable deviations from Newton's Law at sub-millimeter distances. Current experiments investigating short distance gravity can probe extra dimensions too small to be accessible to current collider experiments. In particular for a single extra dimension stabilized by the Casimir energy of the Standard Model fields compactification radii as small as 5 inverse $\mathrm{TeV}$ are accessible to current sub-millimeter gravity experiments.
\end{abstract}




\section{Introduction}

In the last few years theories in which the Standard Model gauge fields propagate in flat extra dimensions at or around the weak scale have attracted considerable interest. For early work on such models see, for example [1] [2] 3]. In some of these theories the Standard Model quarks and leptons are localized to a brane but in others they too propagate in the bulk. Theories of this type have been used to explain, among other things, the fermion mass hierarchy [4], the number of fermion generations [5], the non-observation of proton decay [6], the observed abundance of dark matter [7, the small neutrino mass [8], the weak mixing angle [9] [10] and the origin of supersymmetry breaking (see e.g. [11]). The implications of some of these models for current and future collider experiments have been investigated [12] and used to put bounds on the size of the extra dimensions. The current bound is about 300 $\mathrm{GeV}^{-1}$.

In this paper we consider the problem of stabilizing the volume of the extra dimensions. We find that in a large class of stabilization mechanisms the field which corresponds to fluctuations in the volume of the extra dimensions remains light even after stabilization, and can give sizable corrections to Newtonian gravity. This field is a scalar in the four dimensional effective theory, which we will call the radion. The inverse mass of the radion is typically in the sub-millimeter range. Its couplings violate the equivalence principle, albeit weakly, and mediate a force between matter that is comparable to that of gravity.

The existence of this light field is generic if volume stabilization does not involve dynamics at scales higher than the cutoff of the higher dimensional Standard Model, which we denote by $M_{*}$, and if the effective theory below the compactification scale is four dimensional. The reason is not difficult to understand. Consider a theory where the gauge fields propagate in $D>4$ dimensions. Since the radion, which we denote by $V$, emerges from the higher dimensional gravity sector its action in the four dimensional effective theory has the form below.

$$
\int d^{4} x \sqrt{-g}\left(2 M_{4}^{2} R^{(4)}+\mathrm{c} \frac{2 M_{4}^{2}}{V^{2}} \partial_{\mu} V \partial^{\mu} V-\Omega(V)\right)
$$

Here $\mathrm{c}$ is a dimensionless number of order one that depends on the number of dimensions, $M_{4}$ is the four dimensional Planck scale and $\Omega(V)$ is the potential for the radion. If the dynamics which stabilizes the radion emerges 
from scales lower than or of order the cutoff of the higher dimensional Standard Model then $\langle\Omega\rangle$ is less than or of order $M_{*}^{D}<V>$. We then expect the mass ${ }^{2}$ of the radion to be less than or of order $\frac{M_{*}^{D}<V>}{M_{4}^{2}}$. The requirement that the Standard Model gauge coupling be perturbative up to the scale $M_{*}$ implies that $M_{*}^{D-4}\langle V\rangle$ is bounded from above. We will denote the upper bound on this quantity by $n_{D}$. For five dimensions $n_{5} \approx 740$, while for 6 dimensions $n_{6} \approx 4000$ [14]. For all $D>6 n_{D}<30^{D-4}$. The bound on $n_{D}$ implies that $M_{*}$ is not more than two decades larger than the inverse radius of the extra dimensions if they are toroidal. Since we are assuming that the extra dimensions are at or about the weak scale this then implies a radion mass which is far below the electroweak scale. For example for one extra dimension of inverse radius $500 \mathrm{GeV}$ and the cutoff $M_{*}$ of order 1.5 $\mathrm{TeV}$ we find a radion mass of about $.005 \mathrm{eV}$. A particle of this mass can mediate forces between particles up to a distance of about 40 microns, which is within the range of experiments searching for deviations from Newtonian gravity at short distances. Note that such a small compactification radius is well beyond the range of current collider experiments searching for universal extra dimensions.

In this analysis above we have implicitly assumed that the effective theory below the scale $R^{-1}$ is four dimensional and that the Standard Model along with the radion is a complete description of physics below this scale. If the hierarchy problem is solved at low scales as in the case of large extra dimensions [15] this analysis does not necessarily apply. We will consider this possibility in more detail in a subsequent section.

One may also worry that such a light radion mass would not be stable under radiative corrections, which would raise its mass to the electroweak scale. However this is not the case. The reason is that the radion mass is protected by higher dimensional general covariance, because the radion is part of the higher dimensional gravity multiplet. Hence it is not possible to write a radion mass term in the higher dimensional theory. Therefore the radion mass in the four dimensional effective theory must emerge as a finite effect, and loops which contribute to the radion mass will be cutoff at the compactification scale $R^{-1}$, where $R$ is the radius of the extra dimensions. However since the couplings of the radion in the effective theory are all Planck suppressed the corrections to the radion mass ${ }^{2}$ from quantum effects will also be suppressed by at least two powers of $M_{4}$. Since the only other scale besides $R^{-1}$ available to make up the dimensions of mass ${ }^{2}$ is the cutoff $M_{*}$, 
and because radiative effects are loop suppressed, we do not expect radiative corrections to overwhelm our classical result. For example the correction to the radion mass from the Casimir energy of bulk fields is of order $\frac{1}{16 \pi^{2}} \frac{R^{-4}}{M_{4}^{2}}$ which is much smaller than the typical classical contribution $\frac{M_{*}^{D}<V>}{M_{4}^{2}}$.

From where does the leading correction to Newtonian gravity arise? Consider the coupling of the radion to the $\mathrm{SU}(3)$ gauge fields in the four dimensional effective theory which takes the form

$$
\int d^{4} x \sqrt{-g} \frac{V}{g_{D}^{2}} \operatorname{Tr} F_{\mu \nu} F^{\mu \nu}
$$

Clearly $\sqrt{\frac{g_{D}^{2}}{<V>}}$ is the effective four dimensional $\mathrm{SU}(3)$ gauge coupling $g$ at the matching scale, which is approximately the radius of the extra dimensions, $R=\frac{\leq V>\frac{1}{D-4}}{2 \pi}$. Now the mass of a nucleon is (up to corrections which depend on the light quark massses) proportional to $\Lambda_{Q C D}$, the scale where the $\mathrm{SU}(3)$ gauge coupling becomes strong. Since $\Lambda_{Q C D}$ depends on the radion VEV, the nucleon mass depends on the radion VEV and therefore there is a coupling of the radion to nucleons. The coupling of the radion to the nucleon in linear order

$$
\frac{\partial m_{N}}{\partial<V>}(V-<V>) N \bar{N}=m_{N} \frac{\partial \log \left(\Lambda_{Q C D}\right)}{\partial<V>}(V-<V>) N \bar{N}
$$

Using the renormalization group dependence of $\Lambda_{Q C D}$ on $\langle V\rangle$ we find that the approximate strength of the radion nucleon coupling is given by

$$
\frac{8 \pi^{2}}{9 g^{2}} \frac{m_{N}}{<V>}(V-<V>) \bar{N} N
$$

where $g$ is the strong gauge coupling at the scale $R^{-1}$. Normalizing the radion kinetic term canonically by defining

$$
\Sigma=2 \sqrt{c} \frac{M_{4}}{<V>}(V-<V>)
$$

we see that the coupling of the canonically normalized radion to the nucleon has the form $y_{N} \Sigma \bar{N} N$. From the computation above $y_{N} \approx \frac{3.7}{\sqrt{c}} \frac{m_{N}}{M_{4}}$. However we have ignored the effects of the light quark masses. Including these has the effect of altering $y_{N}$ by a factor of $(1-\chi)$ where $\chi \approx 0.27 \pm .08$ [16]. Therefore our final expression for $y_{N}$ is $(1-\chi) \frac{3.7}{\sqrt{c}} \frac{m_{N}}{M_{4}}$. The radion nucleon coupling 
mediates a potential between matter that has a strength $\frac{y_{N}^{2}}{4 \pi r} \exp \left(-m_{V} r\right)$. Comparing this to the gravitational potential $G_{N} \frac{m_{N}^{2}}{r}$, at short distances the Yukawa force is stronger by a factor $\mathbf{P}=(1-\chi)^{2}\left(\frac{110}{c}\right)$. For one extra dimension $c=\frac{3}{2}$ and the ratio of the radion mediated force to the gravitational force is about $39 \pm 8$. For two extra dimensions $c=1$ and the ratio of the two forces is about $59 \pm 12$. Violation of the equivalence principle is also proportional to the masses of the light quarks, but is highly suppressed [16] and is only at the level of a few parts in a thousand.

In this estimate we have neglected corrections to the four dimensional gauge coupling from higher dimensional operators suppressed by powers of the cutoff $M_{*}$ and from brane localized kinetic and interaction terms. While the effects from higher dimensional operators do not significantly affect the result, the effects of brane localized terms, though volume suppressed, cannot in general be neglected. A quick estimate suggests that the ratio of the contributions to the four dimensional gauge coupling from the bulk terms and the brane localized terms is approximately $\left(\pi R M_{*}\right): 1$ for one extra dimension and $\left(\pi R M_{*}\right)^{2}: 1$ for two extra dimensions. For two extra dimensions the effect of these terms is typically small. However for one extra dimension and $R M_{*}$ of order 3 the corrections to $\mathbf{P}$ are of order 10 percent. The value of $\mathbf{P}$ can be used to distinguish theories with weak scale extra dimensions from other theories that also predict deviations from Newtonian gravity at submillimeter distances. These include large extra dimensions [15, the string dilaton [16] and other string moduli which only get mass after supersymmetry breaking [17] [18] 19] and axions [20]. Weak scale extra dimensions stabilized by supersymmetry breaking and their implications for sub-millimeter gravity experiments have been considered earlier in [21, [22. For a more complete list of theories which predict deviations from Newtonian gravity see [23].

In the following sections we investigate in detail the validity of our conclusions above regarding the the radion mass, which were based on naive scaling arguments. We first consider a specific model of radion stabilization based on the Goldberger-Wise mechanism and show how our conclusions naturally emerge. We then consider the dependence of our results on the manner in which the hierarchy problem is solved. Finally we consider the implications of our result for models in which the radion is stabilized using the Casimir energy of the Standard Model in the bulk, and then we conclude. 


\section{Volume Stabilization}

In this section we consider in detail a simple model with a single compact extra dimension and show how our earlier conclusions naturally emerge once the volume of the extra dimension is stabilized. The compact direction is orbifolded by $S^{1} / Z_{2}$ and there are branes at the orbifold fixed points. We use the Goldberger-Wise [24 mechanism which involves a bulk scalar field with sources on the two branes to stabilize the setup. Our ansatz for the metric is of the form

$$
d s^{2}=g_{\mu \nu}(x) d x^{\mu} d x^{\nu}+r(x)^{2} d \phi^{2}
$$

where $\phi$ is the coordinate along the compact direction and runs from $-\pi$ to $\pi$. It can be shown (see e.g. [25]) that this ansatz reproduces the correct action for the radion in the four dimensional effective theory. The relevant part of the action for our system can be decomposed as

$$
S=S_{G}+S_{M}
$$

where $S_{G}$ and $S_{M}$ are the gravitational and scalar field constituents of the action respectively.

$$
\begin{gathered}
S_{G}=\int d^{4} x d \phi \sqrt{-G}\left(2 M^{3} R^{(5)}-\Lambda_{B}\right)-\delta(\phi) \sqrt{-g} \Lambda_{1}-\delta(\phi-\pi) \sqrt{-g} \Lambda_{2} \\
S_{M}=\int d^{4} x d \phi \sqrt{-G} \frac{1}{2}\left(-\partial_{M} \psi \partial^{M} \psi-m^{2} \psi^{2}\right)+\sqrt{-g} F(\psi) \delta(\phi)+\sqrt{-g} H(\psi) \delta(\phi-\pi)
\end{gathered}
$$

Here $F(\psi)$ and $H(\psi)$ are in general arbitrary functions of $\psi$. For simplicity we will take

$$
\begin{aligned}
& F(\psi)=\lambda_{1} \psi \\
& H(\psi)=\lambda_{2} \psi
\end{aligned}
$$

We obtain an approximate solution for the coupled matter gravity system using the method of Goldberger and Wise. We first solve for the scalar field $\psi$ in the background metric as a function of $r$. We then integrate out the extra dimension to obtain a potential for $r(x)$ which can be used to stabilize the extra dimension. The equation of motion for the scalar field is given by

$$
-r^{-1} \partial_{5} \partial_{5} \psi+m^{2} r \psi=\lambda_{1} \delta(\phi)+\lambda_{2} \delta(\phi-\pi)
$$


The solution (for $\phi>0$ ) has the form

$$
\psi=A \exp (m r \phi)+B \exp (-m r \phi)
$$

where $A$ and $B$ are given by

$$
\begin{aligned}
A & =\frac{1}{2 m} \frac{\lambda_{1} \exp (-m r \pi)+\lambda_{2}}{2 \sinh (m r \pi)} \\
B & =\frac{1}{2 m} \frac{\lambda_{1} \exp (m r \pi)+\lambda_{2}}{2 \sinh (m r \pi)}
\end{aligned}
$$

Substituting the solution for $\psi$ back into the action and performing the integration over the extra dimension we find

$$
S_{M}=\int d^{4} x \sqrt{-g} \frac{1}{4 m}\left(\operatorname{coth}(m r \pi)\left(\lambda_{1}^{2}+\lambda_{2}^{2}\right)+2 \lambda_{1} \lambda_{2} \operatorname{cosech}(m r \pi)\right)
$$

Similarly we substitute the expression for the metric into $S_{G}$ and perform the integration over the extra dimension to obtain

$$
S_{G}=\int d^{4} x \sqrt{-g}\left(4 \pi M^{3} r R^{(4)}-2 \pi r \Lambda_{B}-\Lambda_{4}\right)
$$

with $\Lambda_{4}=\Lambda_{1}+\Lambda_{2}$. We extremize the non-derivative terms in $r$ in the action to find $\langle r\rangle$. At the minimum $\frac{\partial V(r)}{\partial r}=0$ which leads to

$$
\frac{\pi}{4}\left[\left(\lambda_{1}^{2}+\lambda_{2}^{2}\right) \operatorname{cosech}^{2}(m r \pi)+2 \lambda_{1} \lambda_{2} \operatorname{cosech}(m r \pi) \operatorname{coth}(m r \pi)\right]+2 \pi \Lambda_{B}=0
$$

This admits a (local) stable minimum for positive $\Lambda_{B}$ at large $\pi m r \gg 1$ provided $\Lambda_{B} \ll \lambda_{1}^{2}, \lambda_{2}^{2}$ and the product $\lambda_{1} \lambda_{2}$ is negative.

To get the action for four dimensional gravity into the canonical form we perform a Weyl rescaling $g_{\mu \nu} \rightarrow \frac{<r>}{r} g_{\mu \nu}$. Then the gravity action becomes

$$
\int d^{4} x \sqrt{-g} 4 \pi M^{3} r R^{(4)}=\int d^{4} x \sqrt{-g} 2 M_{4}^{2}\left(R^{(4)}+\frac{3}{2 V^{2}} \partial_{\mu} V \partial^{\mu} V\right)
$$

where the radion field $V=2 \pi r$ and the four dimensional Planck scale emerges as $M_{4}^{2}=2 \pi M_{5}^{3}<r>$. After putting the kinetic term for the radion into the canonical form it is straightforward to solve for the mass of the radion.

$$
m_{V}^{2} \approx \frac{m \Lambda_{B} V^{2}}{M_{4}^{2}} \leq \frac{\lambda_{1} \lambda_{2} V}{M_{4}^{2}}
$$


Now if the dynamics which stabilizes the radion does not involve dynamics at scales above the cutoff of the higher dimensional gauge theory $M_{*}$ then $\lambda_{1} \lambda_{2} \leq M_{*}^{5}$ which implies $m_{V}^{2} \leq \frac{M_{*}^{5} V}{M_{4}^{2}}$. This is in perfect agreement with our earlier conclusion which was based on dimensional grounds. However if $\lambda_{1} \lambda_{2} \gg M_{*}^{5}$ then the radion mass can be much heavier than inverse millimeter. We consider the implications of this possibility in the next section.

\section{The Hierarchy Problem}

Under what circumstances is the condition $\lambda_{1} \lambda_{2} \leq M_{*}^{5}$ satisfied? This is related to the manner in which the hierarchy problem is solved. Consider the situation in which the hierarchy problem is solved as in the RandallSundrum model [26]. There is now a warped extra dimension in addition to the compact extra dimension. Models of this type have been considered in [27. We first consider the situation where the scalar field is confined to the brane where the Standard Model fields live. Then the cutoff on the infrared brane $M_{*}$ must be around the weak scale to solve the hierarchy problem, and $\lambda_{1} \lambda_{2} \leq M_{*}^{5}$. Assuming that the radius of the warped extra dimension has been stabilized and has mass of order $M_{*}{ }^{1}$ we can integrate it out to obtain an effective theory which has the same form as in the five dimensional model considered above, and therefore this theory has a light radion. One may worry that mixing between the radion and the excitation corresponding to the radius of the warped extra dimension may change this result but we have checked that such mixing, though present, is too small to affect our conclusions. If however the scalar field lives on the ultraviolet brane then $\lambda_{1} \lambda_{2}$ can be as large as $M_{4}^{5}$ implying a radion much heavier than inverse millimeter. However we see from eqn. (18) that unless the mass $m$ of the scalar field is around the weak scale the size of the extra dimension will tend to be at the Planck scale. This suggests that there is a naturalness problem which needs to be addressed in this framework if the radion is to be much heavier than inverse millimeter.

Now consider the situation in which the hierarchy problem is solved by two or more large extra dimensions of radius much larger than the inverse weak scale. The cutoff of the theory $M_{*}$ must then be at or about the weak scale if the Higgs mass is to be natural. In our model above if the scalar field

\footnotetext{
${ }^{1}$ This will be the case if, for example, it is stabilized using the Goldberger-Wise mechanism.
} 
is localized to the same four brane in the higher dimensional space as the Standard Model fields then $\lambda_{1} \lambda_{2} \leq M_{*}^{5}$ is automatically satisfied. However the form of the radion kinetic term is more complicated because it can mix with other light scalar fields in higher dimensional gravity theory. If there are $\mathrm{N}$ large extra dimensions whose radii $R_{I}(x)$ where $I$ runs from 1 to $\mathrm{N}$ then there are mixing terms of the form $\partial_{\mu} R_{I} \partial^{\nu} r$ in the four dimensional effective theory. However the coefficient of this term before Weyl rescaling is of order

$\frac{M_{4}^{2}}{r R_{I}}$ which is much less than the coefficient of the $\partial_{\mu} r \partial^{\mu} r$ term which is of order $\frac{M_{4}^{2}}{r^{2}}$. Hence the mixing terms are negligible and our conclusions remain unaffected. But what if the scalar field lives in the bulk? Then however a calculation analogous to the one above shows that the radion potential is now of order $V_{N} M_{*}^{D+N} V$ where $V_{N}$ is the volume of the large extra dimensions rather than just $M_{*}^{D} V$. This leads to a radion mass which is much larger than inverse millimeter. Thus it is possible for the radion to naturally be much heavier than inverse millimeter if it is stabilized by dynamics involving the bulk of the large extra dimensions.

\section{Stabilization by Casimir Energy}

Finally for concreteness we consider the implications for short distance gravity experiments of a model with one extra dimension stabilized using the Casimir energy of the Standard Model fields which live in the bulk. Since this effect by itself is not quite sufficient for stabilization we add a brane cosmological constant and bulk cosmological constant ${ }^{2}$.

The contribution to the radion potential from the Casimir effect has been computed 28]

$$
\Omega_{C}(V) \approx-\frac{3 N}{4 \pi^{2}} \frac{1}{V^{4}}
$$

where the number $N$ depends on the number of fields in the bulk and the boundary conditions imposed on them. The contribution to $N$ from a bulk vector field is $3 / 2$ while for a bulk fermion $N=-2$. Every real scalar contributes $1 / 2$ to $N$, while the graviton contributes $5 / 2$. Thus for the bulk Standard Model the total $N \approx-68$. The complete potential including the contributions from the bulk cosmological constant and the brane localized

\footnotetext{
${ }^{2}$ Here we are ignoring the effects of brane localized kinetic terms which we expect to be small.
} 
cosmological constant has the form

$$
\Omega(V)=\Omega_{C}(V)+\alpha V+\beta
$$

Minimizing this potential and requiring that the radion mass be heavier than 50 microns [29] implies that the radius of the extra dimension must be smaller than about $5 \mathrm{TeV}^{-1}$. While this result is sensitive not just to the Casimir energy term but also the forms of additional terms in the radion potential, it shows that in general scales as large as several TeV may be accesible to submillimeter gravity experiments if the leading contributions to the potential arise from Casimir energy.

\section{Conclusions}

We have investigated theories in which the Standard Model gauge fields propagate in extra dimensions at the weak scale and shown that in a large class of models the radion remains light and has a mass in the $10^{-3} \mathrm{eV}$ range. The light radion is characteristic of models in which stabilization does not involve scales larger than the cutoff of the higher dimensional Standard Model, and in which the effective theory below the compactification scale is four dimensional. This is generally the case when the hierarchy problem is solved using an additional warped extra dimension and this class of models will typically have a light radion unless the setup is fine tuned. However this is not the case when the hierarchy problem is solved using large extra dimensions, and in such a setup typically a light radion emerges only if the stabilization mechanism does not involve the bulk of the large extra dimensions.

The light radion mediates an attractive force between matter that is comparable to but somewhat stronger than gravity, and violates the Equivalence Principle albeit by a very small amount. The strength of this force is a characteristic of the number of extra dimensions and is less sensitive to other parameters such as the size and cutoff of the extra dimensions. Current short diatance gravity experiments can probe extra dimensions too small to be visible in collider experiments. In particular compactification radii as small as 5 inverse $\mathrm{TeV}$ are accessible to current experiments if the extra dimensions are stabilized using the Casimir energy of bulk Standard Model fields. We conclude that a unique opportunity exists to explore physics at the weak scale by performing gravitational experiments at the submillimeter scale. 


\section{Acknowledgements}

We would like to thank Eduardo Ponton and Eric Poppitz for discussions at various stages of this work. The authors are supported by the Director, Office of Science, Office of High Energy and Nuclear Physics, of the U. S. Department of Energy under Contract DE-AC03-76SF00098, and by the National Science Foundation under grant PHY-00-98840.

\section{References}

[1] I. Antoniadis, Phys. Lett. B 246, 377 (1990).

[2] I. Antoniadis and K. Benakli, Phys. Lett. B 326, 69 (1994) arXiv:hep-th/9310151.

[3] I. Antoniadis, K. Benakli and M. Quiros, Phys. Lett. B 331, 313 (1994) arXiv:hep-ph/9403290.

[4] N. Arkani-Hamed and M. Schmaltz, Phys. Rev. D 61, 033005 (2000) hep-ph/9903417.

[5] B. A. Dobrescu and E. Poppitz, Phys. Rev. Lett. 87, 031801 (2001) hep-ph/0102010.

[6] T. Appelquist, B. A. Dobrescu, E. Ponton and H. U. Yee, Phys. Rev. Lett. 87, 181802 (2001) hep-ph/0107056.

[7] G. Servant and T. M. Tait, hep-ph/0206071.

[8] R. N. Mohapatra and A. Perez-Lorenzana Phys. Rev. D 66, 035005 (2002)

[9] T. j. Li and W. Liao, Phys. Lett. B 545 (2002) 147 hep-ph/0202090;

L. J. Hall and Y. Nomura, Phys. Lett. B 532, 111 (2002) hep-ph/0202107;

S. Dimopoulos, D. E. Kaplan and N. Weiner, Phys. Lett. B 534, 124 (2002) hep-ph/0202136.

[10] Z. Chacko, L. J. Hall and M. Perelstein, hep-ph/0210149 
[11] A. Delgado, A. Pomarol and M. Quiros, Phys. Rev. D 60, 095008 (1999) hep-ph/9812489;

I. Antoniadis, S. Dimopoulos, A. Pomarol and M. Quiros, Nucl. Phys. B 544, 503 (1999) arXiv:hep-ph/9810410;

R. Barbieri, L. J. Hall and Y. Nomura, Nucl. Phys. B 624, 63 (2002) hep-th/0107004;

R. Barbieri, L. J. Hall and Y. Nomura, Phys. Rev. D 63, 105007 (2001) hep-ph/0011311.

[12] T. Appelquist, H. C. Cheng and B. A. Dobrescu, Phys. Rev. D 64, 035002 (2001) hep-ph/0012100.

[13] A. Albrecht, C. P. Burgess, F. Ravndal and C. Skordis, Phys. Rev. D 65, 123506 (2002) hep-th/0105261.

[14] Z. Chacko, M. A. Luty and E. Ponton, JHEP 0007 (2000) 036 hep-ph/9909248.

[15] N. Arkani-Hamed, S. Dimopoulos and G. R. Dvali, Phys. Lett. B 429, 263 (1998) hep-ph/9803315;

I. Antoniadis, N. Arkani-Hamed, S. Dimopoulos and G. R. Dvali, Phys. Lett. B 436, 257 (1998) hep-ph/9804398.

[16] D. B. Kaplan and M. B. Wise, JHEP 0008, 037 (2000) hep-ph/0008116.

[17] S. Ferrara, C. Kounnas and F. Zwirner, Nucl. Phys. B 429, 589 (1994) [Erratum-ibid. B 433, 255 (1995)] arXiv:hep-th/9405188.

[18] C. Kounnas, F. Zwirner and I. Pavel, standard model," Phys. Lett. B 335, 403 (1994) arXiv:hep-ph/9406256.

[19] S. Dimopoulos and G. F. Giudice, Phys. Lett. B 379 (1996) 105 hep-ph/9602350.

[20] R. Barbieri, A. Romanino and A. Strumia, Phys. Lett. B 387 (1996) 310 hep-ph/9605368.

[21] I. Antoniadis, S. Dimopoulos and G. R. Dvali, Nucl. Phys. B 516 (1998) 70 hep-ph/9710204. 
[22] I. Antoniadis, S. Dimopoulos, A. Pomarol and M. Quiros, Nucl. Phys. B 544, 503 (1999) arXiv:hep-ph/9810410.

[23] J. C. Long, H. W. Chan and J. C. Price, Nucl. Phys. B 539 (1999) 23 hep-ph/9805217.

[24] W. D. Goldberger and M. B. Wise, Phys. Rev. Lett. 83, 4922 (1999) hep-ph/9907447;

W. D. Goldberger and M. B. Wise, Phys. Lett. B 475, 275 (2000) hep-ph/9911457.

[25] M. A. Luty and R. Sundrum, Phys. Rev. D 64, 065012 (2001) hep-th/0012158.

[26] L. Randall and R. Sundrum, Phys. Rev. Lett. 83, 3370 (1999), hep-ph/9905221.

[27] Z. Chacko and A. E. Nelson Phys. Rev. D 62, 085006 (2000) hep-th/9912186.

[28] E. Ponton and E. Poppitz, JHEP 0106, 019 (2001) hep-ph/0105021.

[29] E. G. Adelberger [EOT-WASH Group Collaboration], hep-ex/0202008; J. C. Long, H. W. Chan, A. B. Churnside, E. A. Gulbis, M. C. Varney and J. C. Price, hep-ph/0210004;

J. Chiaverini, S. J. Smullin, A. A. Geraci, D. M. Weld and A. Kapitulnik, hep-ph/0209325 